\title{
Lipoprotein Insulin Resistance Index: A Lipoprotein Particle-Derived Measure of Insulin Resistance
}

\author{
Irina Shalaurova, $\mathrm{PhD},{ }^{1}$ Margery A. Connelly, $\mathrm{PhD},{ }^{1}$ W. Timothy Garvey, $\mathrm{MD},{ }^{2}$ and James D. Otvos, $\mathrm{PhD}^{1}$
}

\begin{abstract}
Background: Lipoprotein particle sizes and concentrations are characteristically altered in patients with insulin resistance (IR) or type 2 diabetes mellitus (T2DM). This study assessed the ability of an IR score, based on nuclear magnetic resonance (NMR)-derived lipoprotein information, to detect IR in otherwise healthy individuals.

Methods: Lipoprotein subclass and size information were evaluated for strength of association with IR, as measured by homeostasis model assessment of insulin resistance (HOMA-IR) in the Multi-Ethnic Study of Atherosclerosis (MESA). To increase the likelihood of identifying subjects with IR, six lipoprotein measures were combined into a single algorithm. The resulting assay [Lipoprotein Insulin Resistance Index (LP-IR)] was developed using HOMA-IR in 4972 nondiabetic subjects from MESA and verified independently using glucose disposal rates (GDRs) measured during hyperinsulinemic-euglycemic clamps in 56 insulin-sensitive, 46 insulin-resistant, and 46 untreated subjects with T2DM.

Results: LP-IR exhibited stronger associations with HOMA-IR $(r=0.51)$ and GDR $(r=-0.53)$ than each of the individual lipoprotein parameters as well as the triglycerides/high-density lipoprotein cholesterol (TGs/HDL-C) ratio $(r=0.41$ and -0.44 , respectively). In MESA, associations between the LP-IR score and HOMA-IR were strong in men $(r=0.51)$, women $(r=0.52)$, European Americans $(r=0.58)$, African Americans $(r=0.48)$, Chinese Americans $(r=0.49)$, and Hispanic Americans $(r=0.45)$. When LP-IR was categorized by HOMA-IR and either body mass index (BMI) or fasting plasma glucose (FPG), subgroups were revealed whose LP-IR scores were high $(\geq 50)$, despite having normal BMIs $\left(<24 \mathrm{~kg} / \mathrm{m}^{2}\right)$ or FPG $(<100 \mathrm{mg} / \mathrm{dL})$.

Conclusions: LP-IR scores had strong associations with multiple measures, HOMA-IR, and GDR, the former being more reflective of hepatic and the latter of peripheral insulin sensitivity, and may represent a simple means to identify individuals with IR.
\end{abstract}

\section{Introduction}

$\mathrm{B}$ EFORE DEVELOPING TYPE 2 DIABETES mellitus (T2DM), individuals pass through two transitional states-a state of insulin resistance (IR) and normoglycemia followed by a state of moderate hyperglycemia termed "prediabetes." IR, also described as IR syndrome, is characterized by decreased tissue sensitivity to the action of insulin, leading to a compensatory increase in insulin secretion. This metabolic dysfunction leads to a cluster of abnormalities with serious clinical consequences, including cardiovascular disease (CVD) and T2DM. ${ }^{1}$ Clinical trials have shown that lifestyle or pharmacological interventions that increase insulin sensitivity or induce weight loss can significantly delay the onset of T2DM in individuals with impaired glucose tolerance. $^{2-5}$ It is conceivable that even earlier intervention applied to insulin-resistant patients, who have not yet experienced loss of $\beta$-cell function and progression to prediabetes, could help prevent, not just delay, development of T2DM. ${ }^{6,7}$

The American College of Endocrinology Task Force on the IR Syndrome agreed that there was clinical value in identifying individuals with IR. ${ }^{1}$ A number of methods are used to assess insulin sensitivity. ${ }^{8}$ The gold standard is the

\footnotetext{
${ }^{1}$ LipoScience Inc., Raleigh, North Carolina.
}

${ }^{2}$ Department of Nutrition Sciences, University of Alabama at Birmingham, and Birmingham VA Medical Center, Birmingham, Alabama. 
glucose disposal rate (GDR) measured during a hyperinsulinemic-euglycemic clamp, which assesses glucose utilization largely by skeletal muscle and adipose tissue and provides a precise measure of insulin sensitivity. ${ }^{9}$ Another means for directly measuring insulin sensitivity is the insulin suppression test from which one can derive steadystate plasma glucose and insulin levels. ${ }^{10-12} \mathrm{~A}$ more indirect method calculates an index of insulin sensitivity, $\mathrm{S}_{\mathrm{I}}$, using the minimal model (MINMOD) analysis of a frequently sampled intravenous glucose tolerance test (FSIVGTT). ${ }^{13}$ All three of these tests require intravenous infusions and are therefore relegated almost exclusively to research settings. Several surrogate indices, including the Matsuda index, are based on dynamic measures of glucose and insulin at different time points during an oral glucose tolerance test. ${ }^{8}$ Because they require several hours of patient monitoring, clinical use is uncommon. Measurements conducted on fasting plasma samples are more amenable for routine clinical use. The most widely used is the homeostasis model assessment of insulin resistance (HOMA-IR), which employs fasting glucose and insulin levels. ${ }^{8}$ Due mainly to biological variability requiring repeat testing and lack of insulin assay standardization, HOMA-IR continues to be used more for research than for clinical applications.

Among the earliest manifestations of IR, observed well in advance of the diagnosis of diabetes or prediabetes, are alterations in lipid and lipoprotein metabolism producing elevations in triglycerides (TGs) and reductions in high-density lipoprotein cholesterol (HDL-C). ${ }^{14}$ McLaughlin and colleagues suggested on this basis that the TGs/HDL-C ratio could provide a simple and clinically accessible alternative to glucose and insulin measurements for identifying insulinresistant overweight and obese patients. ${ }^{15}$ Nuclear magnetic resonance (NMR) spectroscopy provides a more detailed and refined assessment of the lipoprotein abnormalities associated with IR. ${ }^{16-18}$ Specifically, insulin-resistant individuals have higher levels of the large subclass of very-low-density lipoprotein particles (VLDL-P) and the small subclass of lowdensity lipoprotein particles (LDL-P) and lower levels of the large subclass of HDL particles (HDL-P). In addition, mean VLDL particle sizes are generally greater and mean LDL and HDL sizes are smaller in $\mathrm{IR}^{16,17}$ or prediabetic patients. ${ }^{19}$

Clinical NMR testing is increasingly being used to furnish LDL and HDL particle concentrations to aid in CVD risk management. ${ }^{20}$ Because the same NMR measurement simultaneously provides the lipoprotein subclass and size information linked to IR, we sought to develop a composite Lipoprotein Insulin Resistance Index (LP-IR) to assess a patient's IR status. HOMA-IR data from the Multi-Ethnic Study of Atherosclerosis (MESA) was used to guide the development of the LP-IR score, which was then evaluated by comparison to GDR measured in subjects enrolled in a study conducted at the Medical University of South Carolina (MUSC). ${ }^{16,21}$

\section{Methods}

\section{Study populations}

MESA is a large, ongoing observational study comprising 6814 men and women, 45-84 years of age with no evidence of clinical CVD, that was designed to study the prevalence of risk factors for the presence and progression of subclin- ical CVD in a multiethnic cohort. ${ }^{21}$ The protocol was approved by the institutional review board of each of the study centers. Fasting blood samples were collected in EDTA tubes from participants at the baseline exam (2000-2002), and the plasma was stored at $-70^{\circ} \mathrm{C}$. For this particular study, plasma samples were analyzed from 4972 MESA participants who had signed consent forms, had baseline plasma samples available for testing, and did not have diabetes at the start of the study. Lipids, insulin, and glucose measurements were performed at a central location (Laboratory for Clinical Biochemistry Research, University of Vermont, Burlington, VT) using standardized methods and reagents.

The MUSC study population consisted of 148 adult volunteers, with and without T2DM. ${ }^{16}$ Before the study, all patients with T2DM were withdrawn from therapy for at least 3 weeks and followed on an outpatient basis. All subjects were allowed to equilibrate on a weight-maintenance diet $\left(28-32 \mathrm{kcal} \cdot \mathrm{kg}^{-1} \cdot \mathrm{day}^{-1}\right)$ consisting of $50 \%$ carbohydrates, $30 \%$ fats, and $20 \%$ proteins. Subjects were admitted to the General Clinical Research Center, where they remained active, and the isocaloric diet was maintained throughout. Weight had to be stable $( \pm 3 \%)$ for at least 3 months before study, and none of the study subjects engaged in regular exercise. None of the volunteers had cardiovascular, renal, or hepatic disease, and all were chemically euthyroid. No subjects were ingesting any pharmacological agents known to affect carbohydrate homeostasis, lipids, or lipoprotein metabolism. Protocols were approved by the MUSC institutional review board, and written informed consent was obtained from every subject.

\section{Insulin resistance measures}

For MESA study samples, serum glucose was measured on a VITROS analyzer (Johnson \& Johnson Ortho Clinical Diagnostics, Raritan, NJ) using a glucose oxidase method, and insulin was determined by radioimmunoassay using the Linco Human Insulin Specific RIA kit (Linco Research, St. Charles, MO). IR was assessed using homeostasis model assessment of insulin resistance (HOMA-IR) calculated as fasting plasma insulin $(\mu \mathrm{U} / \mathrm{mL}) \times(\mathrm{FPG}[\mathrm{mg} / \mathrm{dL}] \times 0.055) /$ 22.5 , and values were $\log$ transformed for analysis. For the MUSC study, insulin sensitivity was assessed using the euglycemic-hyperinsulinemic glucose clamp technique, as previously described. ${ }^{16,22,23}$

\section{Nuclear magnetic resonance lipoprotein analysis}

Lipoprotein subclass particle concentrations and mean VLDL, LDL, and HDL particle diameters were measured by proton NMR spectroscopy at LipoScience, Inc. (Raleigh, NC) ${ }^{24}$ Amplitudes of the spectroscopically distinct lipid methyl group signals emitted by the different lipoprotein subclasses, derived by deconvolution of the plasma methyl signal envelope using the LipoProfile-3 algorithm, were used to calculate the lipoprotein subclass particle concentrations. Diameter ranges for the pertinent lipoprotein subclasses were as follows: Large VLDL-P>60 nm, small LDL-P 18.0-20.5 nm, and large HDL-P 9.4-14 nm. Mean VLDL, LDL, and HDL particle sizes are weighted averages derived from the sum of the diameter of each subclass multiplied by its relative mass percentage. Within-run and 
between-run LP-IR coefficients of variation, assessed by performing 80 replicate analyses of eight plasma pools over 20 days according to Clinical and Laboratory Standards Institute (CLSI) EP5-A2 guidelines, ${ }^{25}$ were $6 \%$ and $9 \%$, respectively.

\section{Statistical analyses}

Analyses were performed using SAS version 9.2 (SAS Institute, Cary, NC). Because of nonnormal distributions, the analyses used log-transformed values of TGs, the TGs/ HDL-C ratio, large VLDL-P, and HOMA-IR. Two-tailed $P$ values $<0.05$ were considered significant. Spearman rank correlations were used to assess associations of the lipid and lipoprotein parameters with IR calculated by HOMA-IR or GDR. Linear regression analyses with HOMA-IR as the dependent variable were used to assess the relative strengths of association of the six lipoprotein subclass and size parameters with IR, as well as the independence of the relation of LP-IR with IR when adjusted for glucose and body mass index (BMI). Least-squares mean values of HOMA-IR and GDR in LP-IR subgroups, adjusted for age, sex, and race, were calculated using the PROC GLM procedure.

\section{Results}

Clinical and demographic characteristics of the subjects from the MESA and MUSC cohorts are given in Table 1. There were slightly more women than men in both MESA $(52 \%)$ and MUSC (52\%), and the MESA participants were

Table 1. Characteristics of the MESA AND MUSC STUdy PARTICIPANTS

\begin{tabular}{lcc}
\hline Variable & $\begin{array}{c}\text { MESA } \\
(\mathrm{n}=4972)\end{array}$ & $\begin{array}{c}\text { MUSC } \\
(\mathrm{n}=148)\end{array}$ \\
\hline Age, years & $62.0(10.4)$ & $36.9(11.9)$ \\
Women $(\%)$ & 52 & 52 \\
European American $(\%)$ & 42 & 66 \\
Asian American $(\%)$ & 13 & - \\
Hispanic American $(\%)$ & 22 & 3 \\
African American $(\%)$ & 23 & $28.2(5.2)$ \\
BMI $\left(\mathrm{kg} / \mathrm{m}^{2}\right)$ & $28.0(5.3)$ & $120.1(62.9)$ \\
FPG $(\mathrm{mg} / \mathrm{dL})$ & $89.7(10.6)$ & $41.0(13.1)$ \\
HDL-C (mg/dL) & $51.5(14.9)$ & $129(90)$ \\
TGs $(\mathrm{mg} / \mathrm{dL})$ & $128(75)$ & $3.7(3.5)$ \\
TGs/HDL-C ratio & $2.9(2.3)$ & $5.0(5.2)$ \\
Large VLDL-P (nmol/L) & $4.8(6.2)$ & $51.2(7.6)$ \\
VLDL size $(\mathrm{nm})$ & $47.9(8.1)$ & $825(488)$ \\
Small LDL-P $(\mathrm{nmol} / \mathrm{L})$ & $524(379)$ & $4.5(0.7)$ \\
LDL size $(\mathrm{nm})$ & $20.7(0.5)$ & $9.1(0.4)$ \\
Large HDL-P $(\mu \mathrm{mol} / \mathrm{L})$ & $6.1(3.5)$ & $55(21)$ \\
HDL size $(\mathrm{nm})$ & $9.2(0.4)$ & $-11.4(4.2)$ \\
LP-IR score & $43(23)$ & \\
HOMA-IR & $2.1(1.4)$ & \\
GDR & - & \\
\hline
\end{tabular}

Data are shown as mean (standard deviation, SD).

MESA, Multi-Ethnic Study of Atherosclerosis; MUSC, Medical University of South Carolina; BMI, body mass index; FPG, fasting plasma glucose; HDL-C, high density lipoprotein cholesterol; TGs, triglycerides; VLDL-P, very-low-density lipoprotein particle number; LDL-P, low-density lipoprotein particle number; HDL-P, highdensity lipoprotein particle number; LP-IR, lipoprotein insulin resistance index; HOMA-IR, homeostasis model assessment for insulin resistance; GDR, glucose disposal rate. more ethnically diverse (Table 1 ). The six NMR-measured VLDL, LDL, and HDL subclass and size parameters, shown previously to have significant associations with IR, were found to have comparable associations with HOMA-IR in the MESA participants and GDR in the MUSC subjects (Table 2). ${ }^{14,17,26}$ Similar-strength associations were observed between IR and TGs and HDL-C levels, with correlation coefficients ranging generally from about 0.3 to 0.4 .

To take advantage of the simultaneous, automated NMR measurement of the six IR-related lipoprotein subclass and size parameters, with the aim of applying this information clinically to ascertain a patient's IR status, we combined the NMR parameters to produce a multiplex LP-IR score ranging from 0 (most insulin sensitive) to 100 (most insulin resistant). As shown in Table 3, the algorithm used to generate LP-IR divides the six lipoprotein parameters into several particle concentration or size (diameter) categories, assigns each a numerical weighting score, and sums these to produce the LP-IR score. The weighting scores were chosen empirically to reflect the strength and independence of each parameter's association with HOMA-IR in the MESA study population. VLDL size and large VLDL-P were assigned the greatest weighting scores (32 and 22, respectively) followed by HDL size (20), large HDL-P (12), small LDL-P (8), and LDL size (6). We found that combining in this way both the particle size and subclass information, which are not independent of each other and thus somewhat redundant, LP-IR was made more analytically robust and strongly related to IR than by using alternative regression modeling approaches. In addition, capping the weighting scores of large VLDL-P and VLDL size at the high end makes LP-IR less

Table 2. Adjusted Spearman Correlations OF LIPIDS AND NMR LIPOPROTEIN SUBCLASS AND Size Parameters With Different Measures of Insulin Resistance

\begin{tabular}{lrr}
\hline & $\begin{array}{c}\text { HOMA-IR } \\
\text { in } M E S A^{\mathrm{a}}\end{array}$ & $\begin{array}{c}\text { GDR } \\
\text { in }\end{array}$ \\
\hline TGS & 0.36 & -0.47 \\
HDL-C & -0.39 & 0.22 \\
TGs/HDL-C ratio & 0.41 & -0.44 \\
Large VLDL-P & 0.45 & -0.39 \\
VLDL size & 0.40 & -0.42 \\
Small LDL-P & 0.35 & -0.50 \\
LDL size & -0.33 & 0.41 \\
Large HDL-P & -0.40 & 0.47 \\
HDL size & -0.36 & 0.34 \\
LP-IR score & 0.51 & -0.53 \\
\hline
\end{tabular}

Spearman correlations adjusted for age, sex and ethnicity (all $P<0.001)$.

${ }^{a}$ Correlations with HOMA-IR in 4972 participants in the MultiEthnic Study of Atherosclerosis (MESA).

${ }^{\mathrm{b}}$ Correlations with glucose disposal rate (GDR) measured during a hyperinsulinemic-euglycemic clamp ${ }^{16}$ in 148 subjects at the Medical University of South Carolina (MUSC) (46 with untreated T2DM) $(P<0.01$ for HDL-C).

NMR, nuclear magnetic resonance; HOMA-IR, homeostasis model assessment for insulin resistance; MESA, Multi-Ethnic Study of Atherosclerosis; MUSC, Medical University of South Carolina; GDR, glucose disposal rate; TGs, triglycerides; HDL-C, high density lipoprotein cholesterol; VLDL-P, very-low-density lipoprotein particle number; LDL-P, low-density lipoprotein particle number; HDL-P, high-density lipoprotein particle number; LPIR, Lipoprotein Insulin Resistance Index. 
Table 3. LP-IR Score Calculation Algorithm

\begin{tabular}{|c|c|c|c|c|c|c|c|c|c|c|c|}
\hline $\begin{array}{l}\text { VLDL } \\
\text { size } \\
(\mathrm{nm})\end{array}$ & $\begin{array}{l}\text { VLDL } \\
\text { size } \\
\text { score }\end{array}$ & $\begin{array}{c}\text { Large } \\
\text { VLDL-P } \\
(\text { nmol/L) }\end{array}$ & $\begin{array}{c}\text { Large } \\
\text { VLDL-P } \\
\text { score }\end{array}$ & $\begin{array}{l}L D L \\
\text { size } \\
(n m)\end{array}$ & $\begin{array}{l}L D L \\
\text { size } \\
\text { score }\end{array}$ & $\begin{array}{c}\text { Small } \\
\text { LDL-P } \\
(\text { nmol/L) }\end{array}$ & $\begin{array}{c}\text { Small } \\
\text { LDL-P } \\
\text { score }\end{array}$ & $\begin{array}{l}H D L \\
\text { size } \\
(n m)\end{array}$ & $\begin{array}{l}H D L \\
\text { size } \\
\text { score }\end{array}$ & $\begin{array}{c}\text { Large } \\
\text { HDL-P } \\
(\mu \mathrm{mol} / \mathrm{L})\end{array}$ & $\begin{array}{c}\text { Large } \\
\text { HDL-P } \\
\text { score }\end{array}$ \\
\hline$<39.2$ & 0 & $<0.7$ & 0 & $<21.0$ & 6 & $<90$ & 0 & $<8.7$ & 20 & $<3.1$ & 12 \\
\hline $39.2-41.1$ & 1 & $0.7-1.0$ & 2 & 21.0 & 5 & $90-104$ & 1 & 8.7 & 16 & $3.1-4.0$ & 10 \\
\hline $41.2-42.8$ & 2 & $1.1-1.3$ & 5 & 21.1 & 3 & $105-128$ & 3 & 8.8 & 12 & $4.1-5.4$ & 9 \\
\hline $42.8-44.3$ & 4 & $1.4-1.5$ & 7 & 21.2 & 2 & $129-372$ & 4 & 8.9 & 10 & $5.5-6.3$ & 8 \\
\hline $44.4-46.0$ & 6 & $1.6-1.7$ & 9 & $>21.2$ & 0 & $373-961$ & 6 & 9.0 & 9 & $6.4-7.1$ & 6 \\
\hline $46.1-48.1$ & 9 & $1.8-2.5$ & 12 & & & $>961$ & 8 & $9.1-9.2$ & 7 & $7.2-8.0$ & 4 \\
\hline $48.2-50.3$ & 10 & $2.6-3.7$ & 15 & & & & & 9.3 & 5 & $8.1-9.3$ & 2 \\
\hline $50.4-51.6$ & 11 & $3.8-5.3$ & 18 & & & & & $9.4-9.5$ & 4 & $>9.3$ & 0 \\
\hline $51.7-53.2$ & 12 & $5.4-7.9$ & 19 & & & & & $9.6-9.7$ & 2 & & \\
\hline $53.3-55.3$ & 15 & $>7.9$ & 22 & & & & & $>9.7$ & 0 & & \\
\hline
\end{tabular}

$55.4-58.4 \quad 18$

$58.5-61.0 \quad 19$

$61.1-63.0 \quad 22$

$63.1-64.1 \quad 25$

$64.2-65.1 \quad 28$

$>65.1$

Shown are the weighting scores assigned to each category of NMR lipoprotein subclass or size parameter. The LP-IR score is calculated by summing the six NMR parameter scores.

LP-IR, lipoprotein insulin resistance index; VLDL-P, very-low-density lipoprotein particle number; LDL-P, low-density lipoprotein particle number; HDL-P, high-density lipoprotein particle number.

sensitive to genetic or other hypertriglyceridemic states that are not associated with IR.

As indicated in Table 2, the $r=0.51$ correlation of LP-IR with HOMA-IR in the MESA development dataset was stronger than that for TGs/HDL-C $(r=0.41)$, a lipid parameter that has been suggested as a simple means of identifying insulin-resistant patients. ${ }^{15}$ A comparable correlation $(r=-$ 0.53) was observed between LP-IR and the more recognized gold-standard measure of IR, GDR, among the MUSC study participants. This association was also stronger than that between GDR and TGs/HDL-C in this population $(r=-0.44)$.

In MESA, the distribution of LP-IR scores was broad (Fig. 1), reflecting the wide range of insulin sensitivity in this diverse population, and mean levels were higher in men $(48 \pm 23)$ than women $(38 \pm 23)$. Moreover, the LP-IR scores were highly correlated with TGs $(r=0.74)$ and fasting insulin levels $(r=0.51)$ and inversely correlated with HDL-C $(r=-0.67)$. MESA participants divided into 10 subgroups according to LPIR score (e.g., 1-10, 11-20, 21-30, 31-40, etc.) had mean HOMA-IR values (adjusted for age, sex, and race) that were linearly related to LP-IR category (Fig. 2A). The linear relationship between the GDR values and LP-IR scores for individual participants in the MUSC study is shown in Fig. 2B.

Analyses of subgroups of MESA participants revealed very similar relationships between LP-IR and HOMA-IR in men $(r=0.51)$ and women $(r=0.52)$ (Table 4). The LP-IR

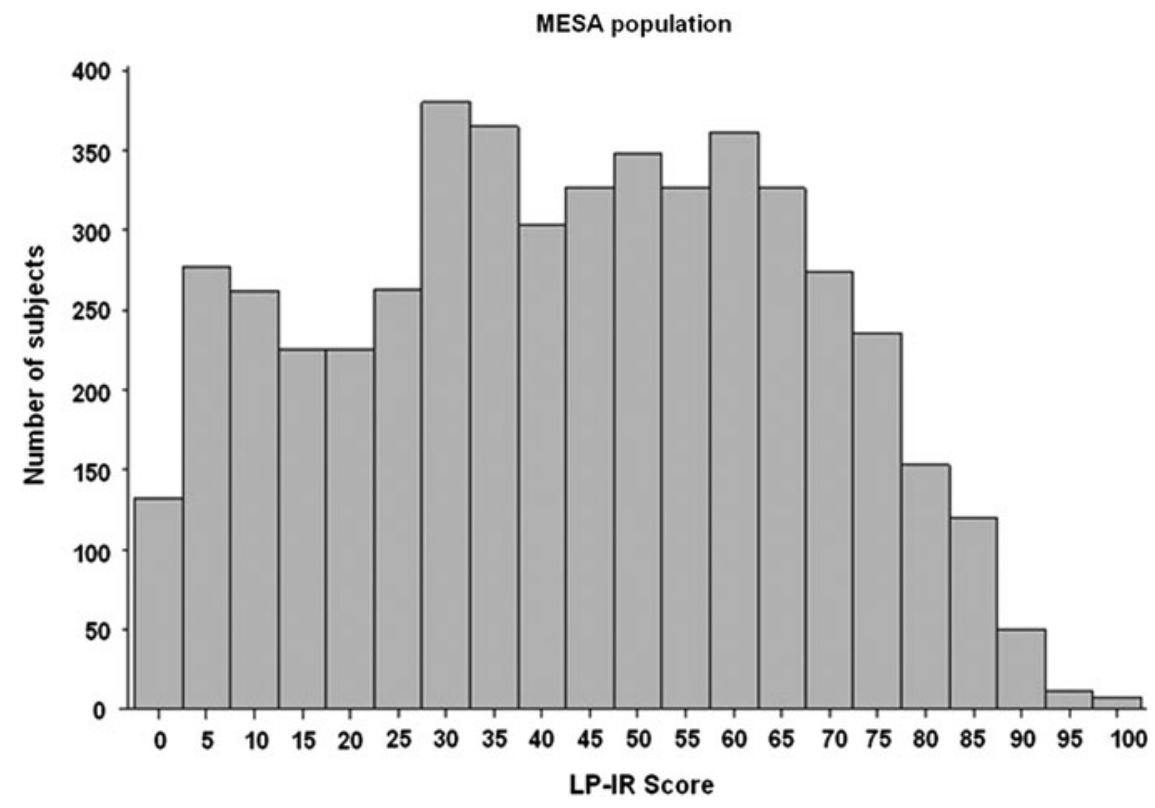

FIG. 1. Distribution of Lipoprotein Insulin Resistance Index (LP-IR) scores in Multi-Ethnic Study of Atherosclerosis (MESA) participants. 

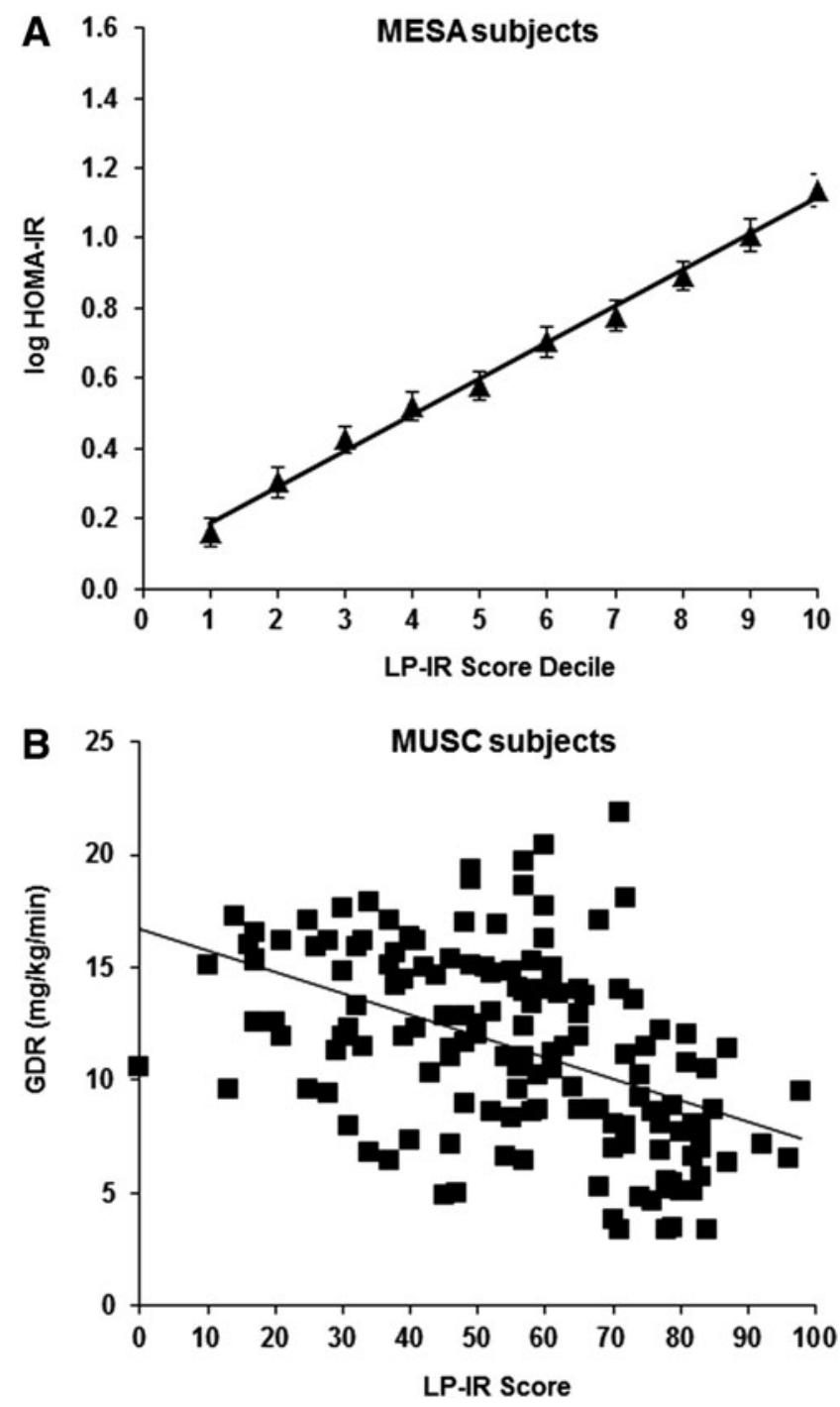

FIG. 2. Relationship between insulin resistance (IR) and Lipoprotein Insulin Resistance Index (LP-IR) score in Multi-Ethnic Study of Atherosclerosis (MESA) and Medical University of South Carolina (MUSC) participants. (A) Least-squares mean and 95\% confidence intervals of logtransformed homeostasis model assessment of insulin resistance (HOMA-IR) in MESA participants adjusted for age, sex, and race in subgroups based on LP-IR score decile. Mean LP-IR scores in deciles 1 to 10 were 5, 16, 27, 34, 42, $50,57,64,71$, and 83, respectively. (B) Scatterplot of glucose disposal rate (GDR) values versus LP-IR scores for MUSC study participants $(n=148)$.

score for European Americans had the strongest association with HOMA-IR $(r=0.58)$. Associations were intermediate in African Americans $(r=0.48)$ and Chinese Americans $(r=0.49)$ and least strong in Hispanic Americans $(r=0.45)$. In all, $14.6 \%$ of MESA participants were taking lipidmodifying therapy, with $13.3 \%$ on statins, $1.1 \%$ on fibrates, and $<0.5 \%$ on either bile acid sequestrants or niacin. Because lipid-altering drugs may alter the relationships between IR and the lipoprotein parameters included in the LP-IR score, we separately examined "untreated" $(n=4186)$ and "treated" $(n=786)$ subjects. The associations of LP-IR with HOMA-IR in these two subpopulations were similar ( $r=0.51$ and $r=0.49$, respectively) (Table 4 ).
The extent to which the LP-IR score detects IR in normal weight and normoglycemic individuals was also examined. Mean HOMA-IR values in MESA were determined in subgroups stratified by BMI and LP-IR as well as FPG and LP-IR. As shown in Fig. 3A, individuals within each BMI category exhibited a range of HOMA-IR values that tracked with LP-IR score (i.e., as HOMA-IR increased, LP-IR score increased). Additionally, there was a clear relationship between BMI and HOMA-IR within each LP-IR category (i.e., as BMI increased, HOMA-IR increased). Interestingly, a subset of individuals with BMI in the normal range $\left(<24 \mathrm{~kg} / \mathrm{m}^{2}\right)$ had elevated LP-IR scores $(\geq 50)$. Similarly, there was a rather large subset of individuals whose LP-IR scores were high $(\geq 50)$ despite having a normal FPG level $(<100 \mathrm{mg} / \mathrm{dL})$ (Fig. 3B). Conversely, there were MESA participants whose BMI was in the overweight/obese range $\left(>25 \mathrm{mg} / \mathrm{kg}^{2}\right)$ or who were prediabetic (FPG $\left.\geq 100 \mathrm{mg} / \mathrm{dL}\right)$, whose LP-IR scores $(<50)$ were low. To further assess the independence of the relation of LP-IR with HOMA-IR in MESA, a linear regression model adjusted for age, gender, and race that included LP-IR, BMI, and FPG was examined. All three parameters added independently to the association with HOMA-IR, giving parameter estimates [per 1 standard deviation (SD)] of $0.23,0.17$, and 0.17 for FPG, BMI, and LP-IR, respectively (all $P<0.0001$ ).

\section{Discussion}

Clinical trials have shown that lifestyle or pharmacological interventions that increase insulin sensitivity with and without weight loss can significantly delay the onset of T2DM. $^{2-5}$ Although there are a number of methods that can be used to assess insulin sensitivity/resistance, ${ }^{8}$ many are costly and time consuming, limiting their use in the primary care setting. A simple and reliable test for assessing IR would potentially enable more effective diabetes prevention by identifying for treatment insulin-resistant patients with normal blood glucose levels and $\beta$-cell function that has not yet deteriorated. ${ }^{27}$ The LP-IR test described here fulfills this need by employing six simultaneously measured NMR lipoprotein subclass and size variables that are each associated with IR. ${ }^{14,17,26}$ The combination of these measures into a single multiplex LP-IR score yielded a parameter exhibiting strong associations with two different research measures of IR, HOMA-IR, and GDR, thereby offering a simple and reliable way to assess a patient's IR status in a clinical setting. Because the information needed to calculate LP-IR is a byproduct of the automated NMR LipoProfile ${ }^{\circledR}$ test that clinicians use to aid patient management of lipoprotein disorders associated with CVD ${ }^{20}$ the LP-IR score can be reported at little or no incremental cost. Recent Food and Drug Administration (FDA) clearance of the highthroughput Vantera ${ }^{\circledR}$ NMR Clinical Analyzer and its intended widespread placement in clinical laboratories should further broaden clinical access to the LP-IR test.

We elected to develop LP-IR on the basis of associations with HOMA-IR rather than with insulin resistance assessed by the gold standard hyperinsulinemic-euglycemic clamp method because clamp data were available for relatively few subjects whereas the much larger MESA HOMA-IR dataset offered a good representation of the full breadth and diversity of the patient population. Given that HOMA-IR is a measure of insulin sensitivity thought to be predominantly 
Table 4. Spearman Correlations of Lipids and NMR Lipoprotein Parameters with Insulin Resistance Assessed by HOMA-IR in Subgroups of the MESA Population

\begin{tabular}{lcccccccc}
\hline & $\begin{array}{c}\text { Men } \\
(\mathrm{n}=2389)\end{array}$ & $\begin{array}{c}\text { Women } \\
(\mathrm{n}=2583)\end{array}$ & $\begin{array}{c}\text { European } \\
\text { American } \\
(\mathrm{n}=2109)\end{array}$ & $\begin{array}{c}\text { Chinese } \\
\text { American } \\
(\mathrm{n}=642)\end{array}$ & $\begin{array}{c}\text { African } \\
\text { American } \\
(\mathrm{n}=1155)\end{array}$ & $\begin{array}{c}\text { Hispanic } \\
\text { American } \\
(\mathrm{n}=1066)\end{array}$ & $\begin{array}{c}\text { Untreated with } \\
\text { lipid drugs } \\
(\mathrm{n}=4186)\end{array}$ & $\begin{array}{c}\text { Treated with } \\
\text { lipid drugs } \\
(\mathrm{n}=786)\end{array}$ \\
\hline TGs & 0.39 & 0.32 & 0.43 & 0.37 & 0.29 & 0.31 & 0.36 & 0.32 \\
HDL-C & -0.35 & -0.43 & -0.43 & -0.40 & -0.30 & -0.33 & -0.39 & -0.33 \\
TGs/HDL-C ratio & 0.42 & 0.41 & 0.50 & 0.43 & 0.35 & 0.37 & 0.43 & 0.37 \\
Large VLDL-P & 0.47 & 0.42 & 0.51 & 0.41 & 0.44 & 0.41 & 0.45 & 0.43 \\
VLDL size & 0.44 & 0.36 & 0.44 & 0.37 & 0.36 & 0.38 & 0.39 & 0.40 \\
Small LDL-P & 0.33 & 0.39 & 0.43 & 0.37 & 0.30 & 0.27 & 0.36 & 0.34 \\
LDL size & -0.31 & -0.35 & -0.42 & -0.39 & -0.27 & -0.26 & -0.35 & -0.34 \\
Large HDL-P & -0.36 & -0.44 & -0.44 & -0.40 & -0.35 & -0.30 & -0.39 & -0.35 \\
HDL size & -0.30 & -0.41 & -0.43 & -0.30 & -0.34 & -0.22 & -0.35 & -0.33 \\
LP-IR score & 0.51 & 0.52 & 0.58 & 0.49 & 0.48 & 0.45 & 0.51 & 0.49 \\
\hline
\end{tabular}

Unadjusted Spearman correlations. All $P<0.0001$.

NMR, nuclear magnetic resonance; HOMA-IR, homeostasis model assessment for insulin resistance; MESA, Multi-Ethnic Study of Atherosclerosis; TGs, triglycerides; HDL-C, high density lipoprotein cholesterol; VLDL-P, very-low-density lipoprotein particle number; LDL-P, low-density lipoprotein particle number; HDL-P, high-density lipoprotein particle number; LP-IR, Lipoprotein Insulin Resistance Index.

related to metabolic changes in the liver as opposed to the periphery (muscle and adipose tissue), ${ }^{8,28}$ it was of interest to determine how well LP-IR reflected the GDR clamp measure of IR, which is driven largely by peripheral insulin sensitivity. ${ }^{8,9}$ The results revealed a strong, linear relationship between the LP-IR score and GDR, indicating that the
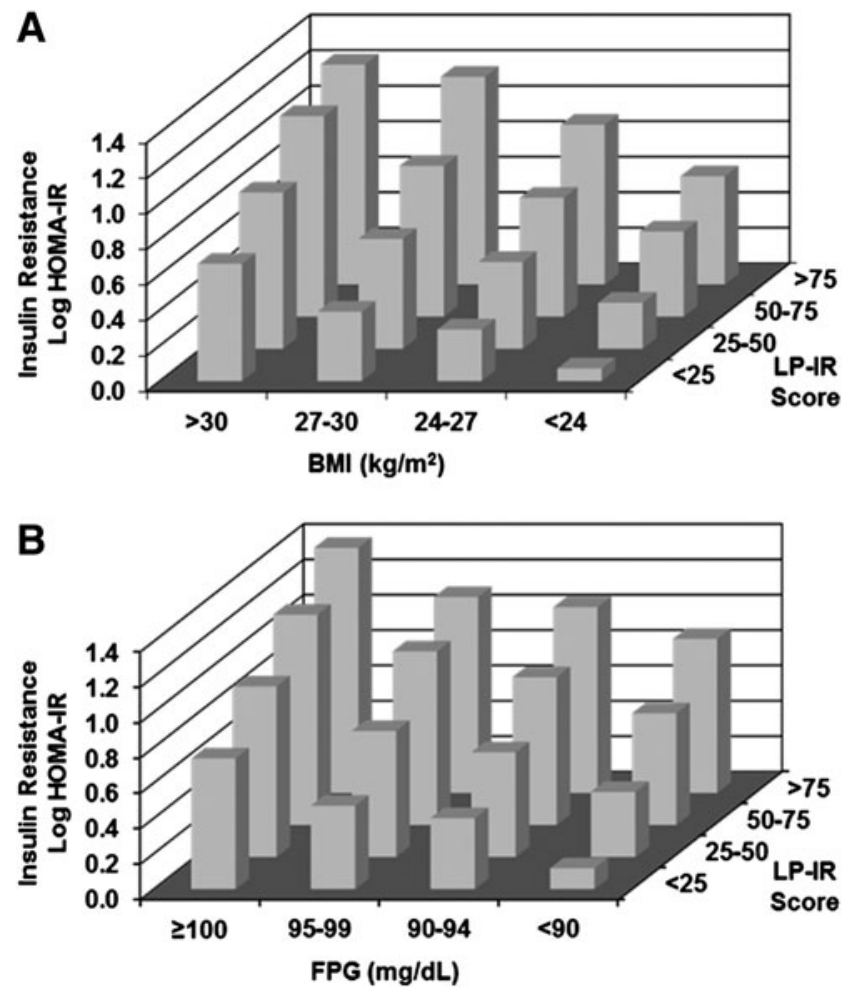

FIG. 3. Insulin resistance as a function of Lipoprotein Insulin Resistance Index (LP-IR) and body mass index (BMI) or glucose in Multi-Ethnic Study of Atherosclerosis (MESA). Least squares mean homeostasis model assessment of insulin resistance (HOMA-IR), adjusted for age, sex, and race, by LP-IR category and body mass index (BMI) (A) and fasting plasma glucose (FPG) (B).
LP-IR assay reflects not only hepatic but also peripheral insulin sensitivity.

It is well known that a subject with a high BMI is more likely to be insulin resistant, although a great amount of individual variance in insulin sensitivity exists independently of generalized adiposity as measured by BMI. ${ }^{29}$ Therefore, the extent to which the LP-IR score detects IR in normal weight individuals was examined. LP-IR was demonstrated to be able to distinguish subjects with IR independent of their BMI. Furthermore, HOMA-IR was more highly correlated with BMI than was the LP-IR score $(r=0.53$ and 0.37 , respectively), suggesting that the LP-IR index is not as dependent on BMI as HOMA-IR for detecting subjects with IR. LP-IR was also shown to be effective in differentiating the IR status of individuals within glucose strata, particularly at the low end of the normal range.

A potential limitation of relying solely on lipoprotein information to identify IR is that results may be compromised by particular lipid-altering medications. Statins, for example, would not be expected to have profound effects on the ability of the LP-IR score to detect IR in a subject with reduced insulin sensitivity because statins have a relatively small effect on the lipoprotein parameters that are heavily weighted in the LP-IR algorithm (i.e., TGs-enriched large VLDL-P, VLDL size, and HDL size). In contrast, niacin treatment, which reduces TGs and increases large HDL-P, ${ }^{30}$ may lower the LP-IR score, giving the illusion of improved insulin sensitivity in a subject who may experience increased IR, ${ }^{31-33}$ $\mathrm{FPG}^{34}$ and risk of progressing to T2DM on niacin therapy. ${ }^{35}$ An analysis of the effects of lipid-modifying therapy on the LP-IR score confirmed that the use of lipid-modifying drugs did not have a substantial effect on the mean LP-IR score in the MESA population. Additional studies comparing the LPIR scores of patients before and after treatment with lipidaltering drugs are required to fully understand the changes that may impact LP-IR assay results and what that would mean to the clinical interpretation of the LP-IR score.

Another potential limitation of the LP-IR index is that it would be reasonable to assume that the lipoprotein parameters that comprise the score will be affected by hypertriglyceridemic conditions that result for reasons other than 
insulin resistance. For example, patients with inherited disorders giving high TGs, such as familial combined hyperlipidemia, may have a high LP-IR score and appear to be insulin resistant in the absence of clinical insulin resistance.

Because IR precedes T2DM, the LP-IR score may also be a convenient and clinically useful predictor of future T2DM, independent of glucose concentration and BMI. In this context, the LP-IR could serve to differentiate future risk of T2DM and help guide the selection and intensity of interventions intended to prevent progression to diabetes as proposed in the complications-centric algorithm for weight loss therapy ${ }^{36}$ by the American Association of Clinical Endocrinologists. ${ }^{37}$ Additional studies need to be performed to compare the LP-IR score with common biomarkers and parameters used for predicting T2DM to prove this is to be the case. However, with the current health care emphasis on preventive medicine, it is intriguing to consider that the LPIR score could be used to alert patients of their heightened risk of developing diabetes even before they become overtly "prediabetic," allowing time for effective lifestyle modification to theoretically prevent, not just delay, onset of the disease.

\section{Acknowledgments}

The authors gratefully acknowledge Drs. Deborah Winegar and Ray Pourfarzib for careful review of the manuscript and scientific comments. This work was supported in part by the Merit Review program of the Department of Veterans Affairs, the National Institutes of Health (DK-038765 and DK-083562), and the UAB Diabetes Research Center (P60-DK079626).

\section{Author Disclosure Statement}

I.S., M.A.C., and J.D.O. are employees of LipoScience, Inc. W.T.G. is an advisor for Alkermes, Plc., DaiichiSankyo, Inc., LipoScience, Inc., VIVUS, Inc., Janssen Pharmaceuticals, Bristol-Myers-Squibb/Astra Zeneca, Eisai, Boehringer-Ingelheim and Novo Nordisk; is a speaker for Merck, Amylin Pharmaceuticals, Inc., and Eisai; is a stockholder for Bristol-Myers-Squibb Company, ISIS/Genzyme, Merck, Pfizer, Inc., Eli Lilly and Company, and VIVUS, Inc.; and has received research support from Amylin Pharmaceuticals, Inc., Merck \& Co., and Weight Watchers International, Inc.

\section{References}

1. Einhorn D, Reaven GM, Cobin RH, et al. American College of Endocrinology position statement on the insulin resistance syndrome. Endocr Pract 2003;9:237-252.

2. Knowler WC, Barrett-Connor E, Fowler SE, et al. Reduction in the incidence of type 2 diabetes with lifestyle intervention or metformin. N Engl J Med 2002;346:393-403.

3. Daniel S, Soleymani T, Garvey WT. A complicationsbased clinical staging of obesity to guide treatment modality and intensity. Cur Opin Endocrinol Diabetes Obes 2013;20:377-388.

4. Torgerson JS, Hauptman J, Boldrin MN, et al. XENical in the prevention of diabetes in obese subjects (XENDOS) study: A randomized study of orlistat as an adjunct to lifestyle changes for the prevention of type 2 diabetes in obese patients. Diabetes Care 2004;27:155-161.
5. Garvey WT, Ryan DH, Henry R, et al. Prevention of type 2 diabetes in subjects with prediabetes and metabolic syndrome treated with phentermine and topiramate extendedrelease. Diabetes Care 2014;37:912-921.

6. American Diabetes Association, National Institute of Diabetes, Digestive and Kidney Disorders. The prevention or delay of type 2 diabetes. Diabetes Care 2002;25:742-749.

7. Genuth S, Kahn R. A step backward-or is it forward? Diabetes Care 2008;31:1093-1096.

8. Muniyappa R, Lee S, Chen H, et al. Current approaches for assessing insulin sensitivity and resistance in vivo: Advantages, limitations, and appropriate usage. Am J Physiol Endocrinol Metab 2008;294:E15-E26.

9. DeFronzo RA, Tobin JD, Andres R. Glucose clamp technique: A method for quantifying insulin secretion and resistance. Am J Physiol 1979;237:E214-E223.

10. Pei D, Jones CN, Bhargava R, et al. Evaluation of octreotide to assess insulin-mediated glucose disposal by the insulin suppression test. Diabetologia 1994;37:843-845.

11. Shen SW, Reaven GM, Farquhar JW. Comparison of impedance to insulin-mediated glucose uptake in normal subjects and in subjects with latent diabetes. J Clin Invest 1970;49:2151-2160.

12. Greenfield MS, Doberne L, Kraemer F, et al. Assessment of insulin resistance with the insulin suppression test and the euglycemic clamp. Diabetes 1981;30:387-392.

13. Bergman RN, Ider YZ, Bowden CR, et al. Quantitative estimation of insulin sensitivity. Am J Physiol 1979;236: E667-E677.

14. Laakso M, Sarlund H, Mykkanen L. Insulin resistance is associated with lipid and lipoprotein abnormalities in subjects with varying degrees of glucose tolerance. Arteriosclerosis 1990;10:223-231.

15. McLaughlin T, Abbasi F, Cheal K, et al. Use of metabolic markers to identify overweight individuals who are insulin resistant. Ann Intern Med 2003;139:802-809.

16. Garvey WT, Kwon S, Zheng D, et al. Effects of insulin resistance and type 2 diabetes on lipoprotein subclass particle size and concentration determined by nuclear magnetic resonance. Diabetes 2003;52:453-462.

17. Goff DC, Jr., D'Agostino RB, Jr., Haffner SM, et al. Insulin resistance and adiposity influence lipoprotein size and subclass concentrations. Results from the Insulin Resistance Atherosclerosis Study. Metabolism 2005;54:264-270.

18. Reaven GM, Chen YD, Jeppesen J, et al. Insulin resistance and hyperinsulinemia in individuals with small, dense low density lipoprotein particles. J Clin Invest 1993;92:141-146.

19. Festa A, Williams K, Hanley AJ, et al. Nuclear magnetic resonance lipoprotein abnormalities in prediabetic subjects in the Insulin Resistance Atherosclerosis Study. Circulation 2005;111:3465-3472.

20. Tamez-Perez HE, Proskauer-Pena SL, Hernrndez-Coria MI, et al. AACE Comprehensive Diabetes Management Algorithm 2013 Endocrine Practice. Endocr Pract 2013; 19:736-737.

21. Bild DE, Bluemke DA, Burke GL, et al. Multi-Ethnic Study of Atherosclerosis: Objectives and design. Am J Epidemiol 2002;156:871-881.

22. Garvey WT, Olefsky JM, Griffin J, et al. The effect of insulin treatment on insulin secretion and insulin action in type II diabetes mellitus. Diabetes 1985;34:222-234.

23. Kennedy A, Gettys TW, Watson P, et al. The metabolic significance of leptin in humans: Gender-based differences in relationship to adiposity, insulin sensitivity, and energy expenditure. J Clin Endocrinol Metab 1997;82:1293-1300. 
24. Jeyarajah EJ, Cromwell WC, Otvos JD. Lipoprotein particle analysis by nuclear magnetic resonance spectroscopy. Clin Lab Med 2006;26:847-870.

25. Clincial and Laboratory Standards Institute. CLSI document EP5-A2: Evaluation of Precision Performance of Quantitative Measurements Methods; Approved GuidelineSecond Edition. Wayne, PA: Clinical and Laboratory Standards Institute, 2004.

26. Goldberg R, Temprosa M, Otvos J, et al. Lifestyle and metformin treatment favorably influence lipoprotein subfraction distribution in the Diabetes Prevention Program. J Clin Endocrinol Metab 2013;98:3989-3998.

27. Frazier-Wood AC, Garvey WT, Dall T, et al. Opportunities for using lipoprotein subclass profile by nuclear magnetic resonance spectroscopy in assessing insulin resistance and diabetes prediction. Metab Syndr Reatl Disord 2012;10:244-251.

28. Pisprasert V, Ingram KH, Lopez-Davila MF, et al. Limitations in the use of indices using glucose and insulin levels to predict insulin sensitivity: Impact of race and gender and superiority of the indices derived from oral glucose tolerance test in African Americans. Diabetes Care 2013;36:845-853.

29. Lara-Castro C, Garvey WT. Diet, insulin resistance, and obesity: Zoning in on data for Atkins dieters living in South Beach. J Clin Endocrinol Metab 2004;89:4197-4205.

30. Davidson MH, Rooney M, Pollock E, et al. Effect of colesevelam and niacin on low-density lipoprotein cholesterol and glycemic control in subjects with dyslipidemia and impaired fasting glucose. J Clin Lipidol 2013;7:423-432.

31. Alvarsson M, Grill V. Impact of nicotinic acid treatment on insulin secretion and insulin sensitivity in low and high insulin responders. Scand J Clin Lab Invest 1996;56:563-570.
32. Kelly JJ, Lawson JA, Campbell LV, et al. Effects of nicotinic acid on insulin sensitivity and blood pressure in healthy subjects. J Hum Hypertens 2000;14:567-572.

33. Poynten AM, Gan SK, Kriketos AD, et al. Nicotinic acidinduced insulin resistance is related to increased circulating fatty acids and fat oxidation but not muscle lipid content. Metabolism 2003;52:699-704.

34. Goldberg RB, Jacobson TA. Effects of niacin on glucose control in patients with dyslipidemia. Mayo Clinic Proc 2008;83:470-478.

35. Group HTC. HPS2-THRIVE randomized placebocontrolled trial in 25673 high-risk patients of ER niacin/ laropiprant: Trial design, pre-specified muscle and liver outcomes, and reasons for stopping study treatment. Eur Heart J 2013;34:1279-1291.

36. Garvey WT. New tools for weight-loss therapy enable a more robust medical model for obesity treatment: Rationale for a complications-centric approach. Endocr Pract 2013;19:864 874.

37. Garber AJ, Abrahamson MJ, Barzilay JI, et al. American Association of Clinical Endocrinologists' comprehensive diabetes management algorithm 2013 consensus statementexecutive summary. Endocr Pract 2013;19:536-557.

Address correspondence to: James D. Otvos, PhD LipoScience Inc. 2500 Sumner Boulevard Raleigh, NC, 27616

E-mail: jotvos@liposcience.com 